\title{
Comparación del grado de especificidad de dos microciclos de entrenamiento en fútbol correspondientes a un equipo profesional y a un equipo en formación \\ Comparing two training microcycles' degree of specificity in professional versus in-formation team \\ *Angel Vales-Vázquez, *AlbertoAreces-Gayo, **Constatino Arce-Fernández, **Julio Torrado-Quintela \\ *Universidad de A Coruña (España), **Universidad de Santiago de Compostela (España)
}

Resumen. En el presente artículo se plantea un estudio de caso descriptivo, en el cual se establece un análisis comparativo del grado de especificidad que presentan dos microciclos de entrenamiento pertenecientes a dos equipos de diferente nivel competitivo (profesional vs. formación). Para ello, se ha construido un novedoso instrumento de evaluación del grado de especificidad que presentan las tareas, sesiones y microciclos de entrenamiento en el fútbol, denominado Escala de Especificidad del Entrenamiento en el Fútbol (E.E.E.F.), fundamentado en una interpretación más amplia y compleja del concepto de especificidad: sincrónica, contextual y funcional (Castelo, 2009; Vales, Arce, Miñano \& García, 2012; Vales 2012). Su estructura interna se configura a partir de cuatro niveles de especificidad: bajo, moderado, elevado y máximo, en base a la aplicación de tres índices de medida originales: Índice de Especificidad de las Tareas (I.E.T.), Índice de Especificidad de las Sesiones (I.E.S.) e Índice de Especificidad de los Microciclos (I.E.M.). Los datos obtenidos ponen de manifiesto que los modelos de entrenamiento aplicados por ambos equipos analizados se desarrollan bajo unas pautas metodológicas caracterizadas por una especificidad media-alta, observándose un mayor grado de especificidad en las tareas, sesiones y microciclos de entrenamiento en el equipo profesional (5,2 puntos $\pm 2,3)$, en comparación al equipo de formación (4,3 puntos \pm 0,4). En su conjunto, los resultados registrados pueden considerarse relevantes para futuros estudios de intervención, así como para el establecimiento de estrategias optimizadoras de los procesos de planificación del entrenamiento en fútbol.

Palabras clave. Fútbol, estrategias de intervención, transferencia entrenamiento-competición.

Abstract. Principio del formulario

This paper presents a descriptive case study, which establishes a comparative analysis of the level of specificity between two microcycles carried out by two teams at different competition level (professional versus in-formation). A new instrument, the Specificity of Football Training Scale (SFTS), was created for assessing tasks specificity, sessions and training microcycles in football. SFTS is based on an broader and more complex interpretation of the concept of specificity: synchronous, contextual and functional (Castelo, 2009; Vales, Arce, Miñano \& García, 2012; Vales 2012). Its internal structure is conformed by four levels of specificity: low, moderate, high and maximum, based on the application of three original measurement indices: Specificity Index of Tasks (SIT), Specificity Index of Sessions (SIS), and Specificity Index of Microcycles (SIM). Data show that training models applied by both teams are developed under methodological guidelines characterized by a medium-high specificity, the professional team showing greater degree of specificity in training tasks, sessions and microcycles (5,2 $\pm 2,3$ points) compared to the in-formation team (4,3 $\pm 0,4$ points). Our overall results can be considered relevant for future intervention studies, as well as for establishing strategies to optimize football training planning processes.

Keywords. Football, intervention strategies, training-competition transfer.

\section{Introducción}

La especificidad del entrenamiento es un principio cada vez más reconocido como prioritario para modelar las respuestas adaptativas del deportista a la competición (Gamble, 2006; Issurin, 2010; Tejada, 2012; Vales, 2012; Vilar, Duarte, Silva, Chow \& Davids, 2014). La evolución experimentada en el conocimiento científico y técnico en el ámbito de las ciencias del deporte y del entrenamiento deportivo en general ha propiciado la aparición de diversas corrientes de pensamiento, inspiradoras de distintas tendencias metodológicas de amplia tradición y aplicación en el entrenamiento de los deportes de equipo (Bayer, 1992; Pacheco, 2004; Stratton, Reilly, Williams \& Richardson, 2004). Vales y Areces (1996); Morcillo, Cano y Martínez (2006); Gil, Moreno, Claver Moreno y Del Villar (2016), apuntan que existen varias teorías relativas a cómo se produce el aprendizaje en el campo de la didáctica de la actividad física y deportiva, destacando como las dos principales opciones las denominadas Teorías conexionistasasociacionistas, en las que el aprendizaje es considerado como un fenómeno orientado al producto, articulado en base a una fuerte conexión y control entre los estímulos propuestos por el entrenador y las respuestas emitidas por el jugador (aprendizaje sin error), y las Teorías cognitivistas-constructivistas, que se refieren a un tipo aprendizajemenos lineal (aprendizaje mediante ensayo-error), en el que se da un valor relevante a los procesos cognoscitivos.

La literatura especializada recoge diversas reformulaciones relativas a los fundamentos teórico-prácticos característicos de la metodología de entrenamiento aplicada en los deportes de equipo en general y en

Fecha recepción: 01-06-16. Fecha de aceptación: 27-10-16 Angel Vales-Vázquez avales@udc.es el fútbol en particular. En su conjunto, todos estos planteamientos metodológicos, elaborados por distintos expertos pertenecientes a diferentes modalidades deportivas y escuelas de entrenamiento (Seiru-lo, 1987; Grosser, Bruggeman \& Zintl, 1989; Antón, 1990; Weineck, 1994; García, Navarro \& Ruíz, 1996; Fradua, 1997; Mombaerts, 2000; Gréhaigne, 2001; Castelo, 2009; Vales \& Areces, 2009; Arjol, 2012), presentan como denominador común un claro interés por responder eficazmente a las necesidades específicas de los deportistas, a partir de la implementación de programas de entrenamiento caracterizados por exhibir estrategias metodológicas con una alta compatibilidad con la propia dinámica de la competición.

En el caso del fútbol, es reconocido por técnicos y especialistas que el éxito deportivo de un equipo dependerá en gran medida de la metodología de entrenamiento que se haya aplicado durante la temporada (Castelo, 2009; Hill-Haas, Couts, Rowsell \& Dawson, 2009; Vales, et all, 2012). Así mismo, actualmente, también es ampliamente aceptado que las diferentes corrientes metodológicas de entrenamiento aplicadas para la optimización del rendimiento de los jugadores han ido evolucionando en el tiempo, detectándose en la práctica la coexistencia de dos tendencias básicas (véase tabal 1), fundamentadas en modelos pedagógicos que podrían considerarse como de naturaleza opuesta. El Paradigma tradicional, basado en teorías de tipo reduccionista y el Paradigma de la complejidad, basado en teorías de corte holístico (Oña, Martínez, Moreno \& Ruíz, 1999; Morín, 2007; Alarcón, Cárdenas, Miranda \& Ureña, 2009).

En el desarrollo las mismas, se pone de manifiesto una visión evolutiva de los diferentes procedimientos metodológicos utilizados en el ámbito del fútbol con el paso del tiempo, caracterizados por dotar de un mayor grado de especificidad formal y funcional a las tareas empleadas durante las sesiones, para provocar adaptaciones óptimas en los jugadores, en función de las exigencias derivadas de la competición. 
I.- Tendencia basada en la fragmentación de los componentes implicados en la respuesta del jugador :

- El proceso de entrenamiento es dividido en contenidos de trabajo cuasi-independientes (físico, técnico, táctico, etc.), sin contemplar

las interrelaciones que conforman y dan sentido al juego.

- El diseño de los medios y métodos de trabajo se produce como producto de una fragmentación de los componentes implicados en las respuestas de los jugadores, para abordarlos de forma autónoma, repetitiva y parcial, esperando que posteriormente, por asociación, se expresen correctamente en la competición.

\section{Corriente Metodológica Integradora}

Se basa en el denominado Paradigma de la Complejidad del proceso de enseñanza-aprendizaje. Parte de una visión global y compleja 等 del juego, que se manifiesta de manera no lineal e imprevisible. Entiende las relaciones entre los participantes y el entorno complejo
del juego como condicionantes fundamentales de la formación del comportamiento competitivo de los jugadores (Gómez, 2011)

I.- Tendencia basada en la yuxtaposición de II.- Tendencia basada en la simulación de III.- Tendencia basada en la simulación de los componentes implicados en la respuesta situaciones variadas del juego: regularidades del sistema de juego del del jugador: - Planteamiento que prioriza una equipo:

- Planteamiento inspirado en las teorías estimulación global del jugador (aspectos - Planteamiento que pretende dotar el sobre el procesamiento de la información perceptivo-decisionales y ejecutivos). entrenamiento de una alta transferencia a la (Singer, 1986). Se parte de un modelo más - La instrumentalización de medios se competición, aportando mayor elaborado del proceso de respuesta del fundamenta en diseñar tareas de tipo global, especificidad al mismo, a partir del diseño jugador, donde, además de la ejecución abierto y con incertidumbre, donde las de tareas que sitúan al jugador en motriz, se tienen en cuenta los componentes relaciones entre los participantes y el contextos de trabajo de alta regularidad y perceptivo- decisionales. entorno variable, pretenden desarrollar en el concreción, fundamentado en las - La instrumentalización de medios se jugador una alta capacidad para adaptarse a particularidades del modelo de juego de su estructura a partir del diseño de tareas por las situaciones cambiantes del juego. niveles de aproximación: general, dirigido, especial y competitivo, con el que se persigue un desarrollo simultaneo de todas las partes tradicionalmente desintegradas.

equipo.

La implementación de medios se basa e una serie de automatismos o rutinas tácticas dirigidas a la interiorización de modelo de juego del equipo. Se enfatiza una dinámica de trabajo que exige al jugador desenvolverse bajo una alta intensidad y concentración.
Partiendo del contexto presentado, en el presente trabajo se plantea como objetivo llevar a cabo un estudio de caso descriptivo, en el que se compara el grado de especificidad que presentan dos microciclos de entrenamiento pertenecientes a dos equipos de diferente nivel competitivo: equipo profesional-élite vs. equipo de formación, a través del análisis de las tareas y sesiones que conforman los mismos.

Para tal fin se ha construido un novedoso instrumento de evaluación de la especificidad que presentan las sesiones y microciclos de entrenamiento, denominado Escala de Especificidad del Entrenamiento en el Fútbol (E.E.E.F.), que se estructura internamente a partir de cuatro niveles de especificidad: bajo, moderado, elevado y máximo, en base a la aplicación de tres índices de medida originales: Índice de Especificidad de las Tareas (I.E.T.), Índice de Especificidad de las Sesiones (I.E.S.) e Índice de Especificidad de los Microciclos (I.E.M.).

Se entiende que los datos obtenidos y procedimientos de evaluación implementados en este estudio de caso, podrán servir como guía a los entrenadores para caracterizar de forma más objetiva el grado de especificidad de los programas de entrenamiento aplicados en sus equipos, constituyendo un contenido interesante para el proceso de optimización de sus metodologías de trabajo.

\section{Método}

\section{Descripción del caso y participantes}

Se trata de un estudio de caso descriptivo, relativo a la evaluación y comparación de la especificidad metodológica que presentan dos microciclos de entrenamiento correspondientes a dos equipos de diferente nivel competitivo, integrados en un mismo club de máximo nivel europeo. Un equipo profesional-élite (Equipo PRO), compuesto por jugadores con una edad media de 26,6 $\pm 1,7$ años, y otro equipo de formación (Equipo FOR), formado por jóvenes jugadores con una edad media de 16,2 $\pm 0,8$ años.

Para unificar los contextos de análisis y evitar la incidencia de ciertas variables sobre las estrategias metodológicas empleadas por ambos equipos en sus rutinas de entrenamiento (e.g. momento de la temporada, densidad competitiva, etc.), se han estudiado dos microciclos ubicados en el denominado periodo competitivo I (primera vuelta del campeonato); ambos bajo un formato de densidad competitiva normal (un partido por semana).

Enla estructura interna delos mismos, cabe destacar queel microciclo correspondiente al Equipo PRO ha constado de cinco sesiones de traba- jo y 22 tareas de entrenamiento (media $=5,1$ ejercicios por sesión), mientras queel microciclo relativo al Equipo FOR ha estado constituido por cuatro sesiones de trabajo en las se aplicaron 17 tareas (media $=4,3$ ejercicios por sesión). Debido al carácter estandarizado y rutinario que presentan las tareas incluidas en las fases de la sesión de calentamiento y vuelta a la calma, en ambos casos se decidió prescindir del análisis las mismas, examinándose únicamente aquellas ubicadas en su parte principal.

Los contenidos de cada uno de los microciclos estudiados han sido facilitados directamente por los cuerpos técnicos de los equipos correspondientes, recogidos en el diario de sesiones de entrenamiento de la temporada, donde se documentan por escrito y gráficamente tanto las tareas realizadas por los jugadores como las condiciones de ejecución de las mismas (duración, repeticiones, etc.). Así mismo, para aclarar posibles dudas sobre la documentación aportada por los equipos, se han mantenido sendas entrevistas con los entrenadores principales de los mismos, donde se detallaron los objetivos y peculiaridades de cada uno de los ejercicios y sesiones desarrollados.

\section{Instrumento de medida}

Para la evaluación del nivel de especificidad de los microciclos objeto de estudio, se plantea una novedosa Escala de Especificidad del Entrenamiento en el Fútbol (E.E.E.F.), estructurada en cuatro niveles de especificidad (véase tabla 2), que permitirá a entrenadores e investigadores en general caracterizar de un modo más objetivo el nivel de especificidad: bajo, moderado, elevado o máximo, que presentan sus entrenamientos.

\begin{tabular}{|c|c|c|c|c|}
\hline Niveles & $\begin{array}{c}\text { Nivel I } \\
\text { Especificidad } \\
\text { Bajo }\end{array}$ & $\begin{array}{c}\text { Nivel II } \\
\text { Especificidad } \\
\text { Moderado }\end{array}$ & $\begin{array}{c}\text { Nivel III } \\
\text { Especificidad } \\
\text { Elevado }\end{array}$ & $\begin{array}{l}\text { Nivel IV } \\
\text { Especificidad } \\
\text { Máximo }\end{array}$ \\
\hline Valores del I.E.S. e I.E.M. & $0-2,24$ & $2,25-4,49$ & $4,50-6,74$ & $6,75-9$ \\
\hline
\end{tabular}

Para la obtención de las puntuaciones que conforman la E.E.E.F., se plantearon tres índices de evaluación de la especificidad que reflejan, tanto las tareas, como las sesiones y microciclos de entrenamiento analizados.

El primero de ellos, denominado Índice de Especificidad de las Tareas (I.E.T.), pretende medir el grado de especificidad que presentan las diferentes tareas pertenecientes a una sesión de entrenamiento, a partir de la aplicación de tres factores determinantes de la magnitud de su especificidad (véase tabla 3): a) Factor I.- Grado de sincronía de los componentes de respuesta del jugador; b) Factor II.- Grado de significatividad contextual de la tarea, y c) Factor III.- Grado de significatividad funcional de la tarea.

Para su cálculo numérico se utilizó una fórmula matemática fundamentada en el sumatorio de las puntuaciones relativas a los tres factores señalados anteriormente, [I.E.T.= puntuación del grado de sincronía de los componentes respuesta del jugador + puntuación del grado de significatividad contextual de la tarea + puntuación del grado de significatividad funcional de la tarea], pudiendo tomar cualquier valor numérico, entero o decimal, comprendido entre 0 (valor mínimo) a 9 (valormáximo).

El segundo de los índices planteados, denominado Índice de Especificidad de las Sesiones (I.E.S.), pretendemedir el nivel de especificidad global de cada una de las sesiones que conforman un microciclo de entrenamiento de un equipo. El I.E.S. representa la medida aritmética de los I.E.T. de la totalidad de tareas que integran cada sesión. Para su 
Tabla 3

Factores, puntuaciones y criterios determinantes del Grado de Especificidad de las Tareas de entrenamiento

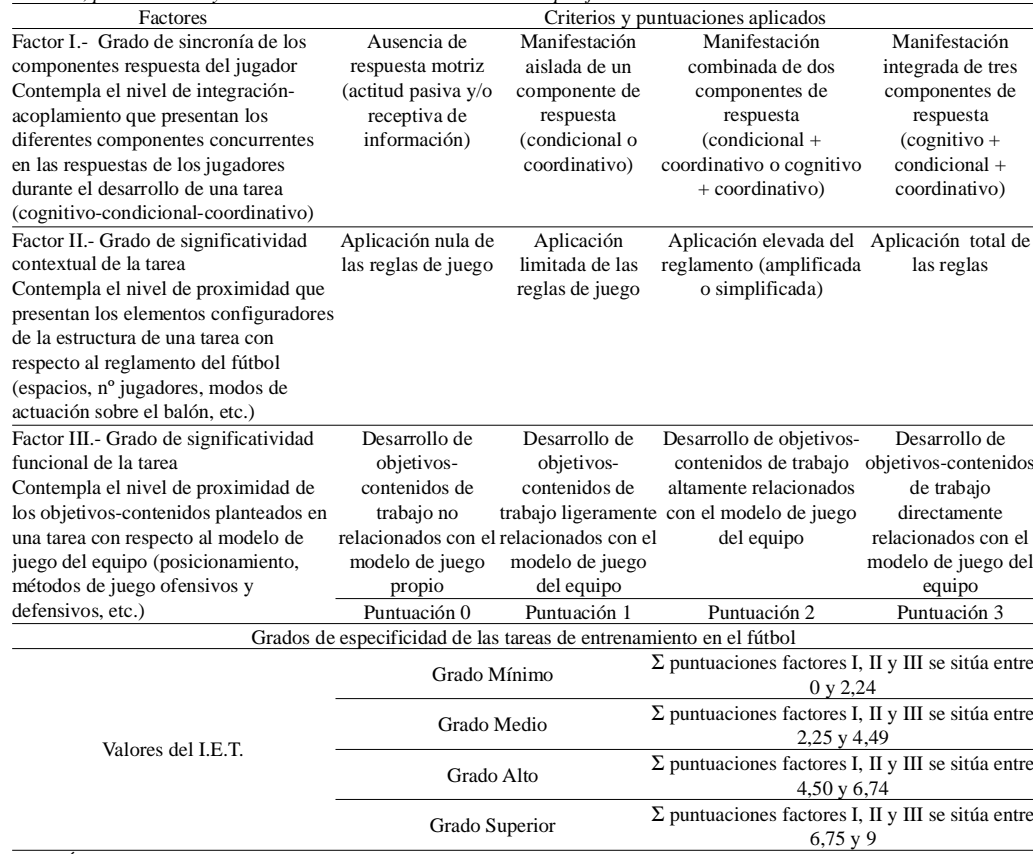

I.E.T.: Índice de Especificidad de las Tareas.

cálculo se utilizóla siguiente fórmula matemática:

[I.E.S. $=\left(\right.$ I.E.T $_{\text {Tarea1 }}+$.E.T. Tarea $2+\ldots+$ I.E.T. Tarea $\left._{\text {n }}\right) / n^{0}$ de tareas de la sesión]

Finalmente, el tercero de los índices utilizados, denominado Índice

de Especificidad de los Microciclos (I.E.M.), pretende medir el nivel de especificidad global de un microciclo de entrenamiento. El I.E.M. supone la medida aritmética de los I.E.S. de la totalidad de sesiones que conforman cada microciclo. Para su cálculo fue utilizada la siguiente fórmula matemática:

[I.E.M. $=\left(\right.$ I.E.S. $_{\text {Sesión1 }}+$ I.E.T. $_{\text {Sesión } 2}+\ldots+$ I.E.T. $\left._{\text {Sesión n }}\right) / n^{\circ}$ de sesiones del microciclo]

Al igual que el I.E.T, tanto el I.E.S. como el I.E.M. podrán tomar cualquier valor numérico, entero o decimal, comprendido entre un rango de puntuaciones que va de 0 (valor mínimo) a 9 (valor máximo) puntos.

Tabla 4

Estructura, contenidos e Índices de Especificidad de las Tareas y Sesiones correspondientes al Microciclo de entrenamiento del Equipo FOR.

\begin{tabular}{|c|c|c|}
\hline Día & Contenido/Actividad & I.E.S. \\
\hline Lunes & Descanso & - \\
\hline Martes & $\begin{array}{l}3 \text { Tareas de trabajo técnico-táctico individual: ruedas de pase-conducción-control } \\
\text { (I.E.T. }=3+3+3 \text { ) } \\
\begin{array}{r}2 \text { Tareas de trabajo táctico-estratégico orientadas a la conservación/recuperación del } \\
\text { balón: posesiones } \\
\text { (I.E.T. }=6+6 \text { ) }\end{array}\end{array}$ & $\begin{array}{c}\Sigma \text { I.E.T. }=21 \\
N^{\circ} \text { tareas: } 5\end{array}$ \\
\hline Miércoles & 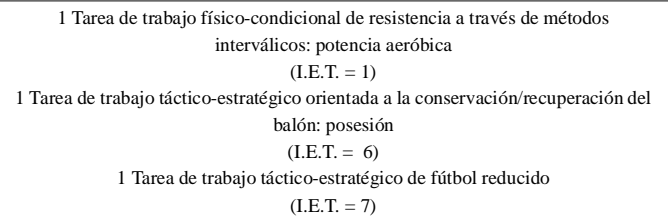 & $\begin{array}{c}\quad 4,7 \\
\sum \text { I.E.T. }=14 \\
\mathrm{~N}^{\circ} \text { tareas: } 3\end{array}$ \\
\hline Jueves & 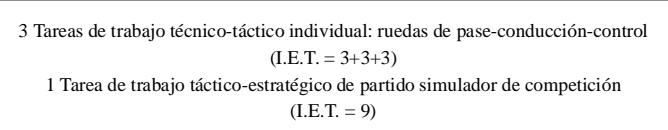 & $\begin{array}{c}4,5 \\
\sum \text { I.E.T. }=18 \\
N^{\circ} \text { tareas: } 4\end{array}$ \\
\hline Viernes & $\begin{array}{l}2 \text { Tareas de trabajo técnico-táctico individual: ruedas de pase-conducción-control } \\
\text { (I.E.T. }=3+3 \text { ) } \\
\begin{array}{r}2 \text { Tarea de trabajo físico-condicional de velocidad integrada } \\
\text { (I.E.T. }=3+3 \text { ) } \\
1 \text { Tarea de trabajo táctico-estratégico de fútbol reducido } \\
\text { (I.E.T. }=7 \text { ) }\end{array}\end{array}$ & $\begin{array}{c}3,8 \\
\sum \text { I.E.T. }=19 \\
N^{\circ} \text { tareas: } 5\end{array}$ \\
\hline Sábado & Descanso & - \\
\hline Domingo & Partido Oficial & - \\
\hline
\end{tabular}

En su interpretación, la obtención de valores bajos en losíndices señalados será indicativo de una utilización de tareas, sesiones y/o microciclos de entrenamiento desarrollados bajo una metodología de trabajo poco específica. Por el contrario, valores altos evidenciarán una aplicación de estrategias metodológicas de entrenamiento muy específicas.

\section{Validez del instrumento de medida.}

Para verificar la validez de contenido de la E.E.E.F. se consultaron diez expertos relacionados con elámbito académico y/o profesional del entrenamiento en fútbol. Se siguieron dos fases. En la primera, fueron informados de los objetivos y aplicaciones del instrumento de medida, así como de la estructura interna y significado de cada uno de los criterios utilizados para medir el grado de especificidad de las tareas de entrenamiento. En la segunda, se les pidió que evaluasen la adecuación de los factores, criterios, puntuaciones e intervalos utilizados para definir el grado de especificidad de las sesiones y microciclos de entrenamiento, contestando a un cuestionario de siete ítems elaborado ad hoc para esta situación. Los datos obtenidos mostraron porcentajes de concordancia superiores al $92 \%$ en todos los casos sometidos a control, indicativos de una validez de contenido satisfactoria del instrumento de medida elaborado.

Complementariamente, con la finalidad de obtener evidencias del grado de fiabilidad del instrumento de medida propuesto, se han llevado a cabo análisis de concordancia intra e inter-observadores a partir del cálculo de los coeficientes de correlación intraclase en una muestra compuesta por 15 tareas de entrenamiento, las cuales superaban ampliamente el 10\% de la muestra total del presente estudio (Tabachimick \& Fidfell, 2007). Para el análisis de la concordancia intra-observador se realizaron dos evaluaciones separadas por cinco días entre sí para evitar la memorización y recuerdo de las valoraciones efectuadas, obteniéndose valores promedios de $.994\left(\mathrm{~F}_{14,14}=157,462, \mathrm{p}\right.$ $<.001)$. Por otra parte, para el análisis de la concordancia inter-observadores, se ha recurrido a la participación de tres especialistas con amplia experiencia en el campo del entrenamiento en el fútbol, a los cuales se les ha explicado previamente el significado de cada uno de los criterios utilizados para medir el grado de especificidad de las tareas de entrenamiento a partir de la E.E.E.F.; los valores promedio obtenidos en este caso fueron de $.992\left(\mathrm{~F}_{14,28}=\right.$ 132,182, $\mathrm{p}<.001$ ).

\section{Aplicación del instrumento de medida.}

Una vez comprobada la validez de contenido y la fiabilidad de medida de la E.E.E.F., se desarrolló un procedimiento de decisión por consenso para determinar las puntuaciones correspondientes a las 39 tareas que configuran los dos microciclos de entrenamiento analizados en el presente estudio. Pare ello, tres entrenadores expertos, atendiendo a los criterios reflejados en la tabla 2, puntuaron conjuntamente cada tarea, según los tres factores de especificidad propuestos anteriormente.

Finalmente, una vez obtenido el valor del I.E.T. para la totalidad de las tareas analizadas, 
Tabla 5 Estructura, conte
Equipo PRO.

\begin{tabular}{|c|c|c|}
\hline Día & Contenido/Actividad & I.E.S. \\
\hline Lunes & 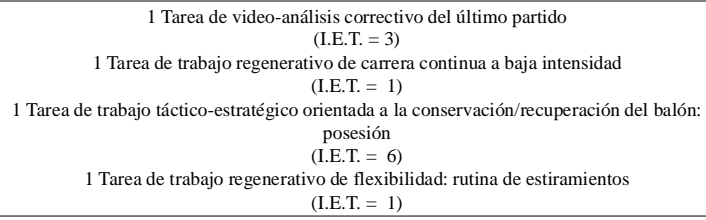 & $\begin{array}{c}2,7 \\
\begin{array}{c}\Sigma \text { I.E.T. }=11 \\
\mathrm{~N}^{\circ} \text { tareas: } 4\end{array}\end{array}$ \\
\hline Martes & Descanso & - \\
\hline Miércoles & 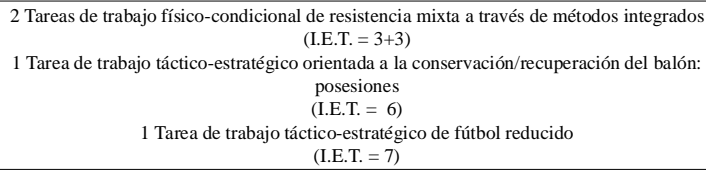 & $\begin{array}{c}4,7 \\
\Sigma \text { I.E.T. }=19 \\
\text { N }^{\circ} \text { tareas: } 4\end{array}$ \\
\hline Jueves & $\begin{array}{l}1 \text { Tarea de trabajo táctico-estratégico de partido simulador de competición } \\
\text { (I.E.T. }=9 \text { ) }\end{array}$ & $\begin{array}{c}9 \\
\Sigma \text { I.E.T. }=9 \\
\mathrm{~N}^{\circ} \text { tareas: } 1\end{array}$ \\
\hline Viernes & $\begin{array}{l}2 \text { Tareas de trabajo físico-condicional de fuerza explosiva integrada } \\
\text { (I.E.T. }=3+3 \text { ) } \\
4 \text { Tareas de trabajo táctico-estratégico de automatismos para combatir/neutralizar equipo } \\
\text { rival } \\
\text { (I.E.T. }=6+6+6+6 \text { ) }\end{array}$ & $\begin{array}{c}5 \\
\Sigma \text { I.E.T. }=30 \\
\mathrm{~N}^{\circ} \text { tareas: } 6\end{array}$ \\
\hline Sábado & $\begin{array}{c}3 \text { Tareas de trabajo físico-condicional de velocidad integrada } \\
\text { (I.E.T. }=3+3+3 \text { ) } \\
3 \text { Tareas de trabajo táctico-estratégico de balón parado ofensivo-defensivo } \\
\text { (I.E.T. }=7+7+8 \text { ) } \\
1 \text { Tarea de trabajo táctico-estratégico de exposición del plan de juego } \\
\text { (I.E.T. }=3 \text { ) }\end{array}$ & $\begin{array}{c}4,8 \\
\Sigma \text { I.E.T. }=34 \\
\text { N }^{\circ} \text { tareas: } 7\end{array}$ \\
\hline Domingo & Partido Oficial & - \\
\hline
\end{tabular}

Estructura, contenidos e Índices de Especificidad de las Tareas y Sesiones correspondientes al Microciclo de entrenamiento del

del entrenamiento administrado a los equipos y jugadores (Mombaerts, 2000; Gréhaigne, 2001; Castelo, 2009; Vales, 2012; Vargas, Urkiza \& Gil, 2015). Del mismo modo, Castelo (2009); Vales (2012); Clemente, Martins y Mendes (2014); Morgans, Orme, Anderson y Drust (2014); Pascual, Alzamora, Carbonell y Pérez (2015), apuntan que en la organización metodológica del entrenamiento, debido a que los procesos de adaptación del jugador presentan un carácter multifactorial y complejo, se hace necesaria la aplicación de sesiones con niveles de especificidad variables, implementadas en función de diversos aspectos que caracterizarán tanto al grupo humano sobre el que se va a intervenir (e.g. edad, experiencias previas, nivel de rendimiento y motivación intrínseca del jugador, etc.), como a los objetivos-contenidos que se pretendan desarrollar en las mismas (e.g. dirigidos al jugador o al equipo, de mantenimiento, de desarrollo o de recuperación, de tipo físico-condicional o técnico-táctico, etc.) y contexto de intervención particular en el que se desenvuelve el proceso de planificación (e.g. densidad de partidos, número de competiciones, etc.).

Los datos obtenidos en el presente estudio evidencian tres cuestiones fundamentales rela-

seprocedióal cálculo de los niveles de especificidad de los dosmicrociclos evaluados, a partir de la aplicación de los I.E.S. e I.E.M. correspondientes.

\section{Resultados}

Tabla 6

Niveles de especificidad de los microciclos de entrenamiento correspondientes a los Equipos FOR. y PRO.

\begin{tabular}{|c|c|c|c|}
\hline & & $\begin{array}{l}\text { Microciclo Equipo } \\
\text { FOR. }\end{array}$ & $\begin{array}{l}\text { Microciclo Equipo } \\
\text { PRO. }\end{array}$ \\
\hline \multicolumn{2}{|c|}{ Niveles de Especificidad del Microciclo } & $\begin{array}{c}\text { Moderado } \\
\text { I.E.M. }=4,3 \pm 0,4\end{array}$ & $\begin{array}{c}\text { Elevado } \\
\text { I.E.M. }=5,2 \pm 2,3\end{array}$ \\
\hline \multirow{4}{*}{$\begin{array}{l}\text { Distribución de las tareas } \\
\text { según su Grado de } \\
\text { Especificidad }\end{array}$} & Grado Mínimo & 1 tarea $/ 5,9 \%$ & 2 tareas $/ 9,1 \%$ \\
\hline & Grado Reducido & 10 tareas $/ 58,8 \%$ & 9 tareas / $40,9 \%$ \\
\hline & Grado Alto & 3 tareas $/ 17,6 \%$ & 5 tareas / $22,7 \%$ \\
\hline & Grado Superior & 3 tareas $/ 17,6 \%$ & 6 tareas / $27,3 \%$ \\
\hline
\end{tabular}

I.E.M.: Índice de Especificidad de los Microciclos.

El presente estudio pretende comparar el grado de especificidad que presentan dos microciclos de entrenamiento pertenecientes a dos equipos de diferente nivel competitivo: Equipo PRO vs. Equipo FOR, utilizando como instrumento de medida la E.E.E.F.

Como puede observarse en la tabla 6, el nivel de especificidad de los dos microciclos analizados se sitúa en una zona intermedia de la E.E.E.F. (niveles II y III). Así, el Equipo FOR presenta un I.E.M. con un valor de 4,3 (nivel moderado), representativo de un uso predominante de tareas en sus sesiones de entrenamiento con un grado de especificidad medio. Por otra parte, también se observa que el Equipo PRO obtiene una puntuación de 5,2 en el I.E.M (nivel elevado), determinado por un uso predominante de tareas con un grado de especificidad alto. En su conjunto, los resultados obtenidos pueden considerarse como relevantes para futuros estudios, así como para el establecimiento de estrategias optimizadoras de los procesos de programación del entrenamiento en el fútbol.

\section{Discusión}

En el ámbito de entrenamiento en el fútbol es ampliamente aceptado que la correcta aplicación de los medios de trabajo (tareas/ejercicios), representa uno de los aspectos que mayor influencia tiene en la calidad cionadas con la definición de estrategias de programación del entrenamiento aplicables, tanto a las etapas de especialización como de rendimiento.

En primer lugar, se observa que los modelos de entrenamiento desarrollados por los dos equipos analizados están caracterizados por una aplicación de tareas con un grado de especificidad medio-alto(I.E.M= 5,2 y 4,3 puntos para el Equipo PRO y Equipo FOR respectivamente), compatibles con los principios orientadores de las tendencias contemporáneas de entrenamiento en el fútbol, en las que se sugiere la conveniencia de instaurar metodologías de entrenamiento con un alto grado de transferencia a la competición (Castelo, 2009; Vales \& Areces, 2009; Gómez, 2011; Arjol, 2012; Clemente, Martins, Mendes \& Figueiredo, 2014). En esta misma línea, Reilly (2005) y Vales et all (2012), apuntan que la gran complejidad de los contenidos a desarrollar, el reducido tiempo entre competiciones y la cada vez mayor importancia del juego colectivo con respecto al individual, determina que el entrenamiento evolucione hacia metodologías basadas en situaciones de juego más específicas.

Como segundo aspecto, cabe destacar que las diferencias detectadas en los niveles de especificidad que presentan ambos microciclos de entrenamiento son reducidas (0,9 puntos), observándose un menor grado en el microciclo correspondiente al Equipo FOR en comparación al Equipo PRO. Esta circunstancia confirma los presupuestos metodológicos establecidos en la Teoría y Práctica General del Entrenamiento Deportivo, en la que se propone una utilización de medios y métodos de entrenamiento con un carácter menos específico en deportistas en periodo de formación con respecto a deportistas de élite(Singer, 1986; Grosser, et all, 1989; Bayer, 1992; Weineck, 1994; Mombaerts, 2000).

Finalmente, como tercera cuestión, los datos obtenidos también reflejan que ambos microciclos presentan, en relación a su estructura interna de contenidos y actividades de trabajo, una distribución de las sesiones de entrenamiento que responde a una dinámica de especificidad de tipo piramidal (creciente - decreciente), caracterizada por concentrar mayores niveles de especificidad de las tareas en las sesiones de entrenamiento coincidentes con los días correspondientes a la parte central del microciclo: 4,6 puntos para el microciclo del Equipo FOR y 7 puntos para el microciclo del Equipo PRO.

En su conjunto, la E.E.E.F. expuesta en el presente estudio, repre- 
senta un instrumento original y útil para clasificar el nivel de especificidad de las diferentes tareas, sesiones y microciclos utilizadas por los entrenadores en sus respectivos programas de entrenamiento.

La presente investigación, no obstante, presenta algunas limitaciones que deberían abordarse en futuros estudios, implementando líneas de trabajo orientadas a verificar la validez empírica (factorial) de la E.E.E.F., a través de su aplicación en contextos reales de entrenamiento más amplios.

\section{Referencias}

Alarcón, F., Cárdenas, D., Miranda, M.T. \& Ureña, N. (2009). Los modelos de enseñanza utilizados en los deportes de equipo. Revista de Investigación Educativa, 13 (23), 101-128.

Antón, J.L. (1990). Balonmano. Fundamentos yetapas de aprendizaje. Madrid: Gymnos.

Arjol, J.L. (2012). La planificación actual del entrenamiento en fútbol: análisis comparado del enfoque estructurado y la periodización táctica. Acción Motriz revista Digital, 8, 27- 37.

Bayer, C. (1992). La enseñanza de los juegos deportivos colectivos. Barcelona: Hispano-Europea.

Castelo, J. (2009). Fútbol: guía práctica de ejercicios de entrenamiento. Barcelona: Paidotribo.

Clemente, F., Martins \& F., Mendes, R. (2014). Periodization based on small-side games: theoretical considerations. Strength and Conditioning Journal, 36(5), 34-43.

Clemente, F., Martins, F., Mendes, R. \& Figueiredo, A. J. (2014). A systemic overview of football game: the principles behind the game. Journal of Human Sport and Exercice, 9(2), 656-667.

Fradua, L. (1997). La visión de juego en el fútbol. Barcelona: Paidotribo.

Gamble, P. (2006). Periodization of training for team sports athetes. Strength and Conditioning Journal, 28(5), 56-66.

García, J.M.; Navarro, M. \& Ruiz, J.A.(1996). Planificación del entrenamiento deportivo. Madrid, Gymnos.

Gil, A., Moreno, P., Claver, F., Moreno, A. \& Del Villar, F. (2016). Manipulación de los condicionantes de la tarea en educación física: una propuesta desde la pedagogía no lineal. Retos. Nuevas tendencias en Educación Física, Deporte y Recreación, 29, $22-27$.

Gómez, P. (2011). La preparación física del fútbol contextualizada en el fútbol. Pontevedra: McSports.

Gréhaigne, J-F. (2001). La organización del juego en el fútbol. Barcelona: Inde.

Grosser, M., Brüggemann, P. \& Zintl, F. (1989). Alto rendimiento deportivo. Barcelona: Martínez Roca.

Hill-Haas, S., Couts, A., Rowsell, G. \& Dawson, B. (2009). Generic versus small-sided game training in soccer. International Journal of Sports Medicine, 30(9), 636-642.

Issurin, V. (2010). New horizons for the methodology and physiology on training periodization. Sports Medicine, 40(3), 189-206.

Mombaerts, E. (2000). Fútbol. Del análisis del juego a la formación del jugador. Barcelona: Inde.

Morcillo, J., Cano, O. \& Martínez, D. (2006). El valor de lo invisible. Fundamentación y propuesta de organización y entrenamiento específico del fútbol. EFDeportes.com Revista Digital, 92.

Morgans, R., Orme, P., Anderson, L. \& Drust, B. (2014). Principles and practices of training for soccer. Journal of Sport and Health Science, 3(4), 251-257.

Morín, E. (2007). Introducción al pensamiento complejo. Barcelona: Ed. Gedisa S.A.

Oña, A., Martínez, M., Moreno, F. \& Ruíz, L.M. (1999). Control y aprendizaje motor. Madrid. Síntesis.

Pacheco, R. (2004). Fútbol. La enseñanza y el entrenamiento del fútbol 7. Barcelona: Paidotribo.

Pascual, M., Alzamora, E., Carbonell, J.A. \& Pérez, J.A. (2015). Análisis de los diferentes métodos de enseñanza utilizados en el fútbol base. Retos. Nuevas tendencias en Educación Física, Deporte y Recreación, 28, 94-97.

Reilly, T. (2005). Training specificity for soccer. International Journal of Applied Sports Sciences, 17(2), 17-25.

Seiru-lo, F. (1987). Opciones de planificación en los deportes de largo periodo competitivo. Revista RED, vol. I, 3, 53-62.

Singer, R.(1986). El aprendizaje de las acciones motrices en el deporte. Barcelona: Hispano Europea.

Stratton, G., Reilly, T., Williams, A.M. \& Richardson, D. (2004). Touth soccer. From science to performance. Londres: Routledge.

Tabachnick, B. \& Fidell, L. (2007). Using multivariate statistics. Boston, United States: Allyn and Bacon.

Tejada, C. (2012). Efecto del entrenamiento mediante el método comprensivo en ultimate frisbee. Revista Internacional de Medicina y Ciencias de la Actividad Física y el Deporte, vol. 12 (46), 329-348.

Vales, A. \& Areces, A. (1996). Propuesta organizativa de las perspectivas de análisis de los deportes de equipo. Revista RED. Revista Entrenamiento Deportivo, tomo X, 3, 35-44.

Vales, A. \&Areces, A. (2009). La planificación del entrenamiento en los deportes de equipo: bases conceptuales y estado de la cuestión. Revista RED. Revista Entrenamiento Deportivo, tomo XXIII, 1, 514.

Vales, A., Areces, A., Miñano, J. \& García, J.A. (2012). Propuesta de un modelo para la caracterización de las sesiones de entrenamiento en el fútbol de rendimiento. Revista Fútbol P.F., 3, 34-53.

Vales, A. (2012). Fútbol: del análisis del juego a la edición de informes técnicos. Pontevedra. Mc Sports.

Vargas, A., Urkiza, I. \& Gil, S. (2015). Efectos de los partidos de pretemporada en la planificación deportiva: variabilidad en las sesiones de entrenamiento. Retos, 27, 45-51.

Vilar, L., Duarte, R., Silva, P., Chow, J.Y., \& Davids, K. (2014). The influence of pitch dimensions on performance during small-side and conditioned soccer games. Journal of Sports Science, 32(19), 1751-1759.

Weineck, J. (1994). Fútbol total. Barcelona: Paidotribo.
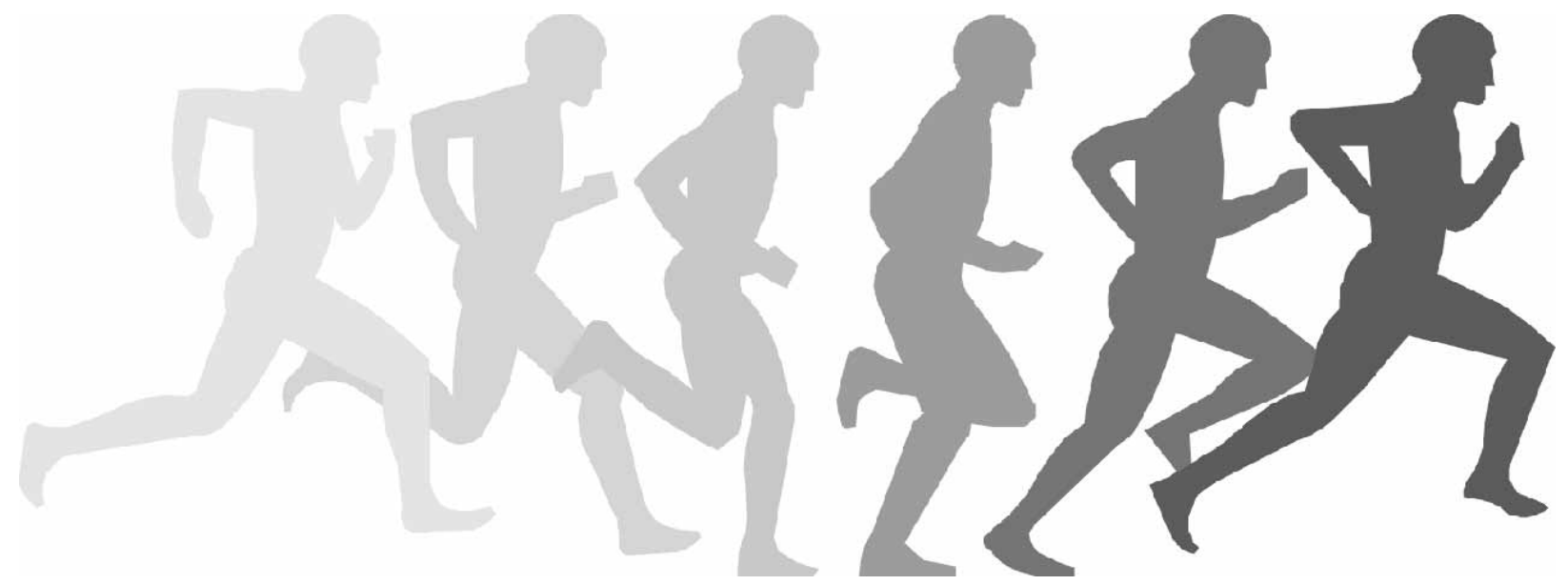